\title{
Pulmonary hyperclarity pattern in torax radiography: associated pathologies
}

\section{Patrón de hiperclaridad pulmonar en radiografía de tórax: patologías}

\section{asociadas}

Karla E. Abundiz-Bibiano ${ }^{1 *}$, Roberto Sotelo-Robledo ${ }^{2}$, and Juan M. Chino-Mendoza ${ }^{3}$

${ }^{1}$ Service of Diagnostic and Therapeutic Imaging, Escuela en Posgrados de Sanidad Naval, Universidad Naval, Secretaria de Marina; ${ }^{2}$ Department of Radiology, Instituto Nacional de Enfermedades Respiratorias. Mexico City, Mexico ; ${ }^{3}$ Service of Radiology, Hospital El Alto Sur, La Paz, Bolivia

\section{ABSTRACT}

The presence of the hyperclarity pattern in chest radiography studies (chest X-RAY), like other radiological patterns, guides us to reduce our differential diagnoses, this coupled with the clinical picture, age of the patient and suspicion of delivery. Demonstrating that in certain pathologies, the simple radiographic study continues to be of great help, correlating with tomographic studies when necessary.

Key words: Chest X-Ray. Pulmonary hyperclarity. Thorax tomography.

\section{RESUMEN}

La presencia del patrón de hiperclaridad en los estudios de radiografía de tórax (rayos $X$ de tórax), asi como otros patrones radiológicos, nos ayuda a reducir los diagnósticos diferenciales, esto aunado al cuadro clínico, la edad del paciente y la sospecha diagnostica. Demostrando que en ciertas patologías, el estudio radiográfico simple de torax sigue siendo de gran ayuda, correlacionando con estudios tomográficos en algunas ocasiones cuando es necesario.

Palabras clave: Radiografia de torax. Hiperclaridad pulmonar. Tomografia de torax. 


\section{INTRODUCTION}

There are different radiological patterns that can be identified by chest radiography, which help us reduce our differential diagnoses when we know how to identify them properly, in the same way when they are applied to the clinical context of each patient.

The pattern of pulmonary hyperclarity in thorax radiography is an example of which, with an adequate interpretation of this finding, plus some other associates, makes us suspect in pathology oriented pulmonary destruction as the first option.

\section{EMPHYSEMA}

The term emphysema is coined with the abnormal enlargement of the air spaces distal to the terminal bronchiole coupled with the destruction of the walls of these airspaces ${ }^{1}$. In imaging studies, for example chest radiography, there are some findings that can make us infer this pathology, since the diagnosis is not made directly by this imaging technique ${ }^{2}$.

Through tomography, it is divided as follows, according to its findings (Fig. 1).

\section{CENTRILOBULAR EMPHYSEMA}

Centrilobular emphysema is characterized by areas of low attenuation observed by a high resolution tomography study, which lack a visible wall, with a greater predominance towards the upper lobes, in radiographic study one can suspect the finding of areas of increased radiolucence. This type of emphysema is associated

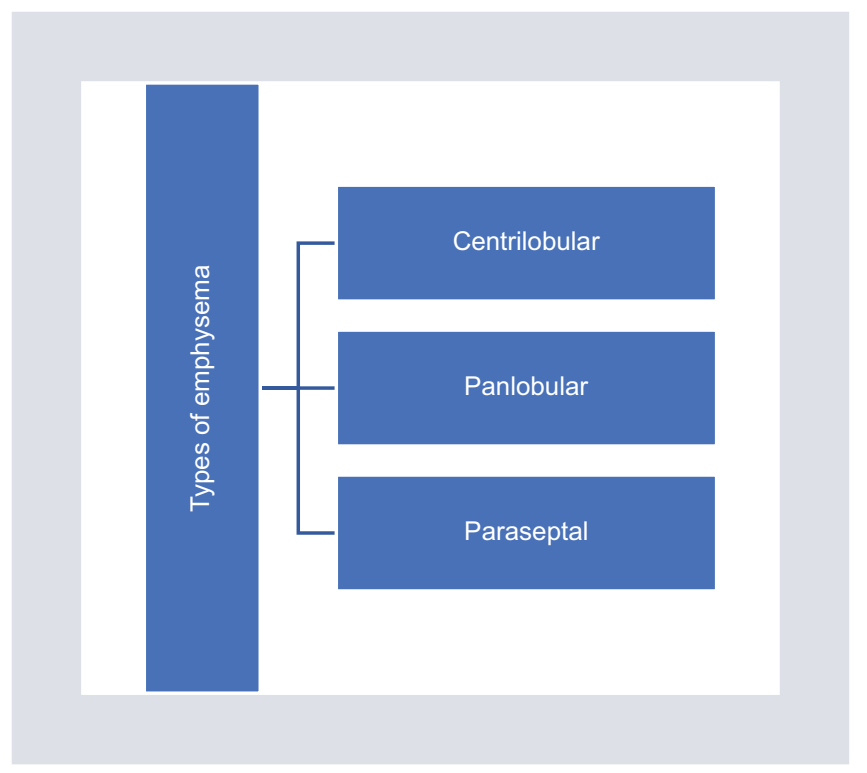

Figure 1. Types of emphysema.

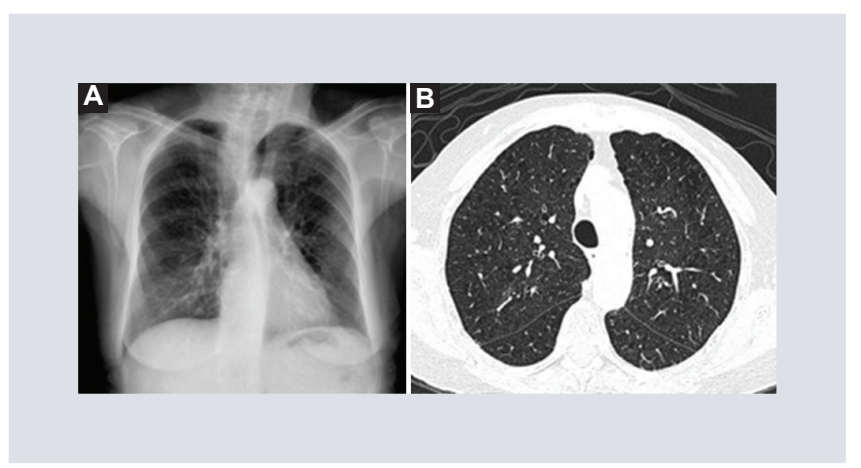

Figure 2. A: chest x-ray of the anterior posterorax and chest tomography in the lung window in a 55-year-old male patient with a history of smoking, where a pattern of hyperclarity toward the upper lobes of both lungs predominates, corroborated by tomography. B: where centrilobular emphysema is observed.

in a greater percentage to patients with a history of smoking ${ }^{3}$ (Fig. 2).

\section{PANLOBULAR EMPHYSEMA}

This term is mainly used for tomography findings in patients with alpha - 1 antitrypsin deficiency, since it is rare to find it in those who consumed cigarettes ${ }^{4}$. There are other 


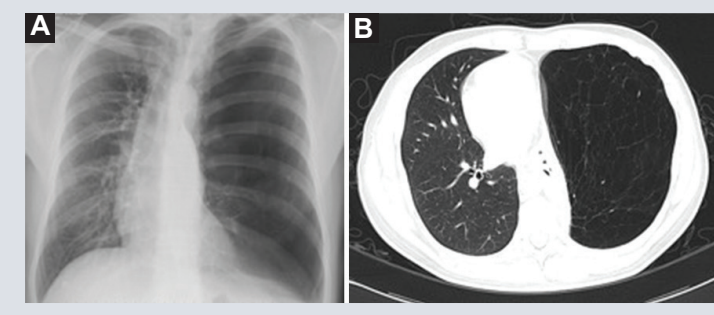

Figure 3. A: anterior posteroral chest radiograph and axial window pulmonary tomography of a 15 -year-old male patient with a diagnosis of congenital emphysema. $\mathbf{B}$ : in which the asymmetry is shown by a larger area of left pulmonary hyperclarity associated with the increase in intercostal spaces that correlates in the tomography by areas of low density with partially visible wall.

associated pathologies such as Swyer-James Syndrome and the use of Ritaling, however it can be observed in other processes that involve permanent destruction of the acini distal to the respiratory bronchiole.

By radiography, some of the suggestive findings are pulmonary hyperclarity, increased anterior antero diameter, retro sternal and caudal skull of the thorax, as well as flattening of the diaphragm, increased intercostal space. In the tomography study which is where suspicion is verified, low attenuation areas are displayed homogeneously towards the lower lobes (Fig. 3).

\section{PARASEPTAL EMPHYSEMA}

Like centrilobular emphysema, paraseptal emphysema, is associated with the history of cigarette consumption, this due to the increase in pulmonary macrophages and neutrophils, producing destruction with the formation of enlarged airspaces, both patterns may coexist along with other alterations ${ }^{5}$.

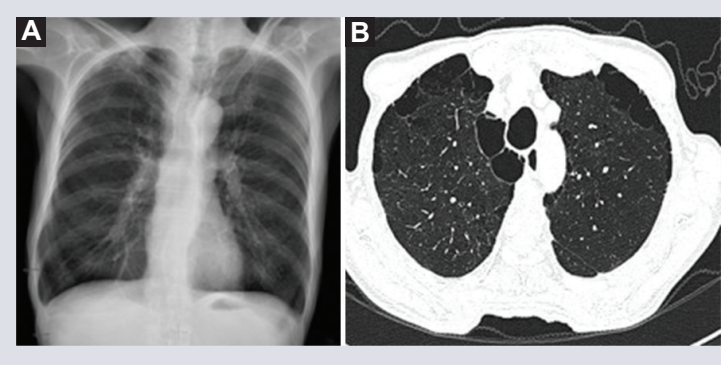

Figure 4. A: chest radiograph and tomography with window for lung of a 52-year-old male patient, with a history of cigarette consumption, in which some areas of hyperclarity or hyper lucenic are identified at the level of upper lobes without visible wall. B: which in tomography are correlate with low density areas of anterior paraseptal predominance.

In a study of simple thoracic radiology, as in the other types of emphysema, they are inferred by areas of hyper clarity without visible walls, however in paraseptal emphysema, these are adjacent to the pleura. In the tomographic study, the suspicion is corroborated by areas of low attenuation adjacent to the pleura of up to 10 $\mathrm{mm}$. On some occasions it must be differentiated with the Honey comb pattern (Fig. 4).

\section{PNEUMOTHORAX}

The term pneumothorax is used to define the existence of air or gas between the visceral and parietal pleura, which can enter through the chest wall or through the pulmonary parenchyma ${ }^{6}$. This can be sub-divided according to its cause into (Fig. 5).

In turn, spontaneous pneumothorax is subdivided into primary (without lung disease) and secondary (lung disease present).

On the chest X-ray, pneumothorax is suspected in the presence of greater hyper clarity or 


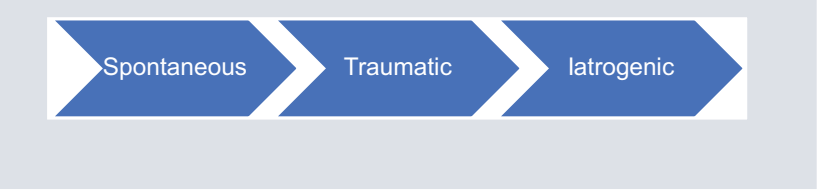

Figure 5. Types of pneumothorax according to the cause.

hyper lucidity towards the periphery, compared to the lung against the lateral, as well as the identification of the visceral pleural border. On some occasions, the support of a lateral projection and maximum expiration is necessary.

As for the tomography study, it can be requested when it comes to progression of a primary disease in a secondary spontaneous pneumothorax, as well as when there is doubt in simple radiography studies ${ }^{7}$, in which we can find free air in the Pleural cavity and the extent of it can be delimited more accurately, with this plus the clinical context, define treatment (Fig. 6).

\section{ASTHMA}

It is considered a chronic disease that affects the respiratory tract, causing inflammation and changes in their normal structure. The diagnosis is clinical and through bronchodilator spirometry ${ }^{8}$.

The symptoms (cough, wheezing and dyspnea) can develop from childhood before 5 years in $80 \%{ }^{9}$, however the diagnosis is made at later ages.

Chest $\mathrm{x}$-ray, there are no characteristic signs and may even become normal, or enter areas

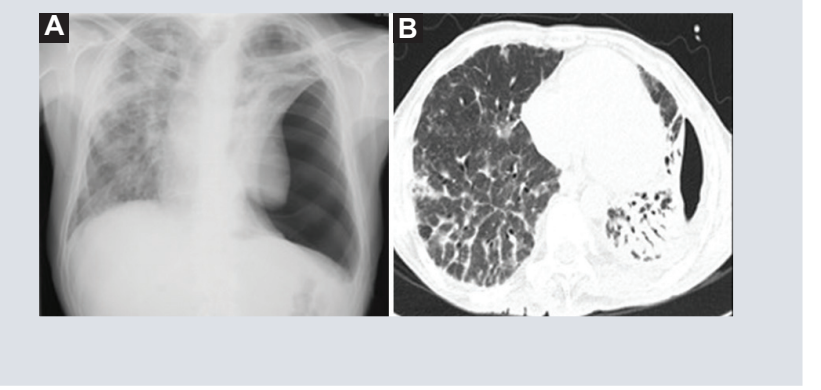

Figure 6. A: a 67-year-old male with a diagnosis of diffuse interstitial disease, in which a chest $x$-ray with pulmonary asymmetry is observed at the expense of hyper clarity of the left hemi thorax towards the middle and lower lobe due to pleural displacement, same as tomography. B: atelectasis is identified by compression secondary to spontaneous pneumothorax.

of hyperlucides and increased intercostal space.

The tomographic study is useful to rule out some complications that may be associated with asthma, not for a direct diagnosis. Among the findings, we can find (Fig. 7):

- Air trapping in expiratory phase.

- Reduction of attenuation in inspiratory phase.

- Thickening of the bronchial wall.

- Centrolobular opacities.

\section{BULLA}

It is defined as an air space $>1 \mathrm{~cm}$ with a thin wall $<1 \mathrm{~mm}^{10}$ (Fig. 8).

There are different pathologies that can be associated with this finding, the most common is chronic obstructive pulmonary disease, however it can be found in the normal lung in $20 \%$ and $80 \%$ of the emphysematous lung parenchyma ${ }^{11}$ (Fig. 9) 


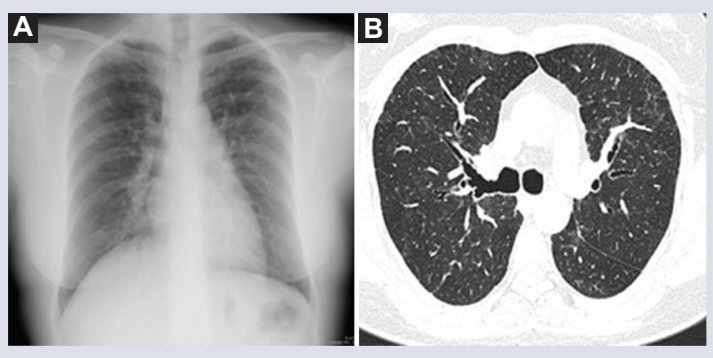

Figure 7. A: a 23-year-old female patient with a diagnosis of asthma, in which a chest $\mathrm{x}$-ray with areas of hi perclarity is observed at the level of the upper lobes of both lungs, this comparatively with the lower and middle lobes. B: which in tomography study are visualizes attenuation decrease in inspiratory phase.

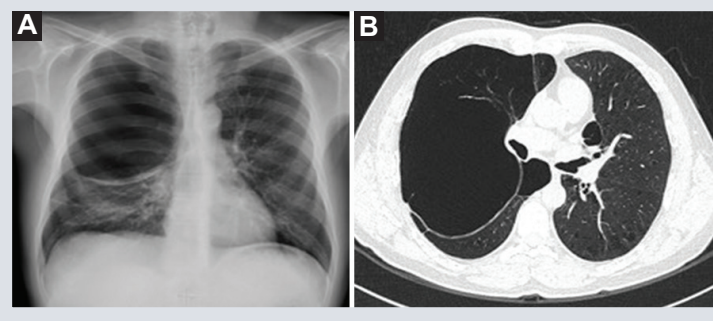

Figure 9. A: a 63-year-old male patient with a diagnosis of emphysema and a history of smoking, with a chest x-ray and tomography, in which there is a hyper-clear area at the level of the upper and middle lobes of the right lung. $\mathbf{B}$ : with a thin wall by tomography.

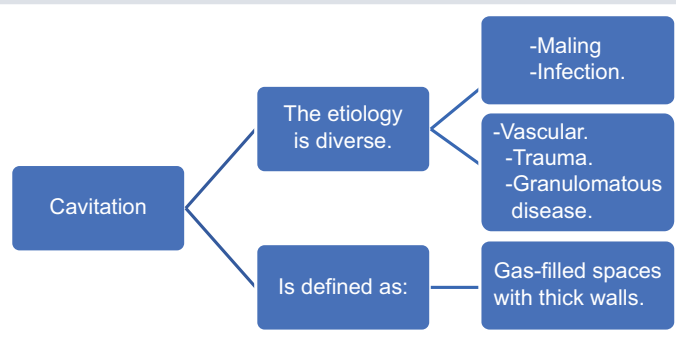

Figure 10. Cavitation, etiology and definition.

tomography, which helps us assess the extent of the disease (Fig. 11).

\section{SWYER - JAMES SYNDROME}

\section{CAVITATION}

Of the causes associated with cavitations, the most frequent is related to infectious processes, specifically post-primary tuberculosis, with a typical presentation of involvement in the upper lobes up to $85 \%{ }^{12}$ (Fig. 10).

Cavitation can be identified by imaging studies such as chest radiography, as well as
This syndrome is considered the most common cause of unilateral pulmonary hyper lucidity, as a result of post infectious bronchiolitis obliterans ${ }^{13}$.

Through imaging studies, 1 or more affected lung lobes and even both lungs can be observed. In the radiographic study, pulmonary asymmetry is suspected due to hyper clarity 


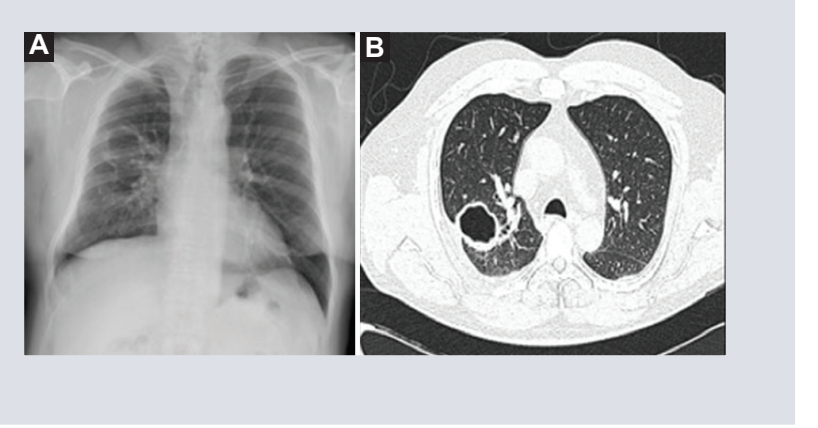

Figure 11. A: a 38-year-old female with a diagnosis of pulmonary tuberculosis, in which we observed a radiograph of the anterior posteroral thorax, with a hyperclarity image, which shows a thick comparison located in the upper lobe of the right lung. B: one that corroborates by tomography.

of some of the lungs in a comparative way, the definitive diagnosis is made by clinical correlation and with tomographic study the extent of the disease (Fig. 12).

There are different differential diagnoses, such as foreign body aspiration and congenital lobar emphysema, which can be ruled out by means of targeted tomography techniques ${ }^{14}$.

\section{COMBINED SÍNDROME}

The so-called combined syndrome is characterized by the presence of emphysema associated with pulmonary fibrosis, the latter is known as a specific form of fibrosing interstitial pneumonia with chronic and progressive behavior $^{15}$.

This combination is associated with a smoking rate of more than 30 packages per year ${ }^{16}$. Among the imaging studies, high resolution tomography plays an important role since it shows the involvement of the

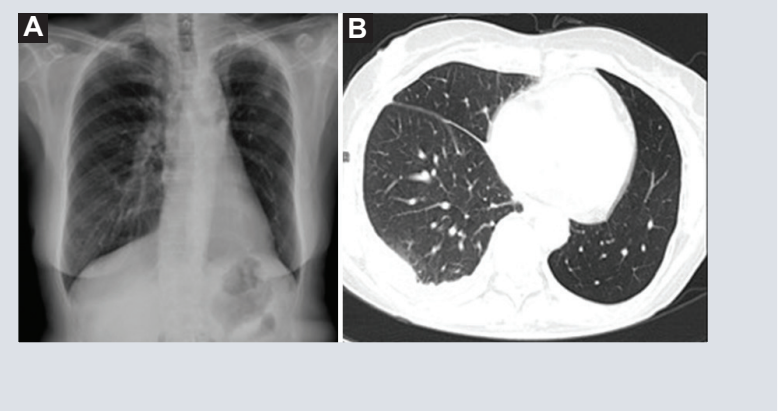

Figure 12. A: chest radiography and thoracic tomography in a 16-year-old female patient, where we observed thoracic asymmetry due to greater hyper clarity of the left lung that corresponds to areas of hypo-attenuation by tomography. B: characteristic of Swyer-James syndrome.

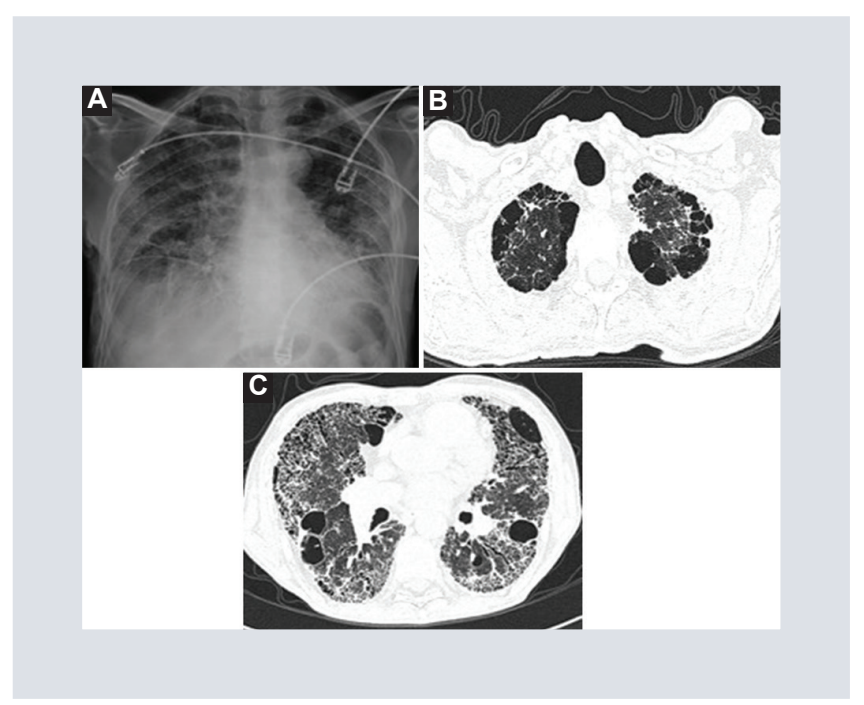

Figure 13. A: a 69-year-old male patient with a diagnosis of pulmonary fibrosis and a chest x-ray of the anteropositor, which shows an increase in the hyper clarity of predominance towards the upper lobes, associated with diffuse reticular pattern.

B-C: which in a tomography study correspond to paraseptal emphysema and honey comb.

lung parenchyma, in the simple radiographic study you can visualize areas of greater hyper lucidity by areas of emphysema, considering the suspicion of this syndrome when interstitial disease pattern is added (Fig. 13). 


\section{CONCLUSION}

- The diagnoses associated with the hyperclarity or hyper lucidity pattern are extensive, however we must take into account the patient's clinical context, as well as the type of population or background to reduce our diagnostic suspicion.

- The hyperclarity pattern can affect a lung lobe or both lungs, with this we can reduce our suspicion, it can also be shown as cyst, cavitation or bull.

- In the context of unilateral involvement, we must keep in mind the pneumothorax, Swyer james syndrome, congenital lobar emphysema and bronchial aspiration, in bilateral involvement the emphysema associated with smoking is one of the first options.

- It should be mentioned that the simple radiographic study may be limited by its twodimensional acquisition, the tomographic study being helpful to assess the extent of some pathologies and characterization of doubtful lesions by radiography.

\section{CONFLICT OF INTERESTS}

The authors declare not to have any interest conflicts

\section{ETHICAL DISCLOSURES}

Protection of human and animal subjects. The authors declare that no experiments were performed on humans or animals for this study.
Confidentiality of data. The authors declare that they have followed the protocols of their work center on the publication of patient data.

Right to privacy and informed consent. The authors declare that no patient data appear in this article.

\section{REFERENCES}

1. Kemp SV, Polkey MI, Shah PL. The Epidemiology, Etiology, Clinical Features, and Natural History of Emphysema. Thorac Surg Clin. 2009; 19(2):149-58

2. Murphy A, Danaher L. Pulmonary emphysema. Radiopaedia. 2014.

3. Webb WR, Muller NL. High-resolution CT of The Lung. Lippincott Williams \& Wilkins, a Wolters Kluwer business. Philadelphia. USA, 2018.

4. Lynch DA, Moore CM, Wilson C, et al. CT-based Visual Classification of Emphysema: Association with Mortality in the COPDGene Study. Radiology. 2018;288(3):859-66.

5. Grenier PA. Relationship between Interstitial Lung Abnormalities and Emphysema in Smokers with and Those without COPD. Radiology. 2018;288:610-1.

6. Daley BJ. Pneumothorax. Medscape. 2018.

7. Swierzy M, Helmig M, Ismail M, Rückert J, Walles T, Neudecker J. Pneumothorax. Zentralbl Chir. 2014;1:S69-86.

8. Tseng H-J, Henry TS, Veeraraghavan S, Mittal PK, Little BP. Pulmonary Function Tests for the Radiologist. Radiographics. 2017;37:1037-58.

9. Darras KE, Roston AT, Yewchuk LK. Imaging Acute Airway Obstruction in Infants and Children. Radiographics. 2015;35:2064-79.

10. Hansell DM, Bankier AA, MacMahon H, McLoud TC, Muller NL, Remy J. Fleischner Society: Glossary of Terms for Thoracic Imaging. Radiology. 2008;246:697-722.

11. Saínz Menéndez B. Enfisema pulmonar y bullas de enfisema. Clasificación. Diagnóstico. Tratamiento. Rev Cubana Cir. 2006;45(3-4).

12. Nachiappan AC, Rahbar K, Shi X, et at. Pulmonary Tuberculosis: Role of Radiology in Diagnosis and Management. Radiographics. 2017;37:52-72.

13. Milliron B, Henry TS, Veeraraghavan S, Little BP. Bronchiectasis: Mechanisms and Imaging Clues of Associated Common and Uncommon Diseases. Radiographics. 2015;35:1011-30

14. Dillman JR, Sanchez R, Ladino-Torres MF, Yarram SG, Strouse PJ, Lucaya J. Expanding upon the Unilateral Hyperlucent Hemithorax in Children. Radiographics. 2011;31:723-41.

15. Raghu G, Remy-Jardin M, Myers JL, Richeldi L, Ryerson CJ, Lederer DJ, et at. Diagnosis of Idiopathic Pulmonary Fibrosis. An Official ATS/ERS/ JRS/ALAT Clinical Practice Guideline. Am J Respir Crit Care Med. 2018;1;198(5):e44-e68.

16. Mejía M, Buendía-Roldán I, Mateos Toledo H, Estrada A, EspinozaHernández M, Juárez-Hernández F. Primer Consenso Mexicano sobre Fibrosis Pulmonar Idiopática. Neumol Cir Torax. 2016;1:32-51. 